\title{
The Density and the Velocity Power Spectrum in Thermally Bi-stable Flows
}

\author{
Adriana Gazol ${ }^{1}$, and Jongsoo Kim ${ }^{2}$ \\ ${ }^{1}$ Centro de Radioastronomía y Astrofísica UNAM, \\ A. P. 3-72, c.p. 58089, Morelia, Michoacán, México \\ email: a.gazol@crya.unam.mx \\ ${ }^{2}$ Korea Astronomy and Space Science Institute, \\ 61-1, Hwaam-Dong, Yuseong-Ku, Daejeon 305-348, Korea \\ email: jskim@kasi.re.kr
}

\begin{abstract}
We present numerical results concerning the behavior of the density and the velocity power spectrum in turbulent thermally bistable flows for different Mach numbers.
\end{abstract}

Keywords. turbulence, instabilities, ISM: kinematics and dynamics, ISM: structure

The turbulent nature of the interstellar medium (ISM) is believed to play a crucial role on the determination of its density and velocity structure (e.g. Elmegreen \& Scalo 2004; Scalo Elmegreen (2004) ). In the atomic interstellar gas, turbulence coexists with the isobaric mode of thermal instability (TI) (Field 1965), which do also play a crucial role on the determination of the structure and the dynamics of this kind of gas. The density and the velocity spectra of the atomic gas are thus the result of the interplay between these two physical ingredients.

In this paper we present results from a numerical study on the behavior of the density and the velocity power spectra in thermally bistable flows. We have analyzed a set of five 3D simulations where turbulence is randomly driven in Fourier space at a fixed wavenumber and with different Mach numbers $M$ (with respect to the gas at $10 \mathrm{~K}$ ) ranging from 0.2 to 4.5. For details concerning the numerical model see (Gazol \& Kim 2010).

The density power spectrum becomes shallower as $M$ increases (see fig. 1). This behavior is the same as the one reported for isothermal simulations (Kim \& Ryu 2005), however the slope values we obtain are much shallower than those obtained for the isothermal case. This fact and the trend we observe are interpreted as a consequence of the simultaneous turbulent compressions, thermal instability generated density fluctuations, and the weakening of thermal pressure force in diffuse gas. This behavior is consistent with the fact that observationally determined spectra exhibit different slopes in different regions. The values of the spectral indexes resulting from our simulations are consistent with observational values. For a detailed discussion on the interpretation as well as on comparison with observations, see (Gazol \& Kim 2010).

We do also explore the behavior of the velocity power spectrum, which becomes steeper as $M$ increases (see figure 2), and which for low values of $M$ is also shallower than the one obtained for isothermal simulations. The spectral index goes from a value much shallower than the Kolmogorov one for $M=0.2$ to a value steeper than the Kolmogorov one for $M=4.5$. The flattening of the spectrum for low $M$ could be due to the increased relevance of the velocities generated by the development of TI, but a detailed analysis of the velocity power spectrum for thermally bistable flows is needed. For a discussion on this issue see (Gazol \& Kim 2010). 


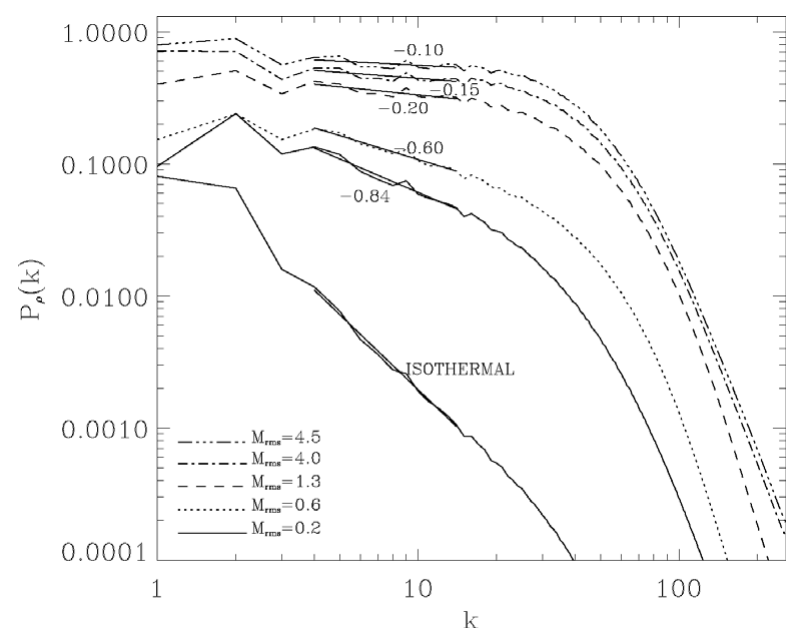

Figure 1. Density power spectra $P_{\rho}$ for simulations with different $M$. For $M=0.2$ the spectra resulting from an isothermal run is also displayed. Solid lines represent least-squares fits over the range $4 \leqslant k \leqslant 14$ and numbers indicate the slopes of these fits.

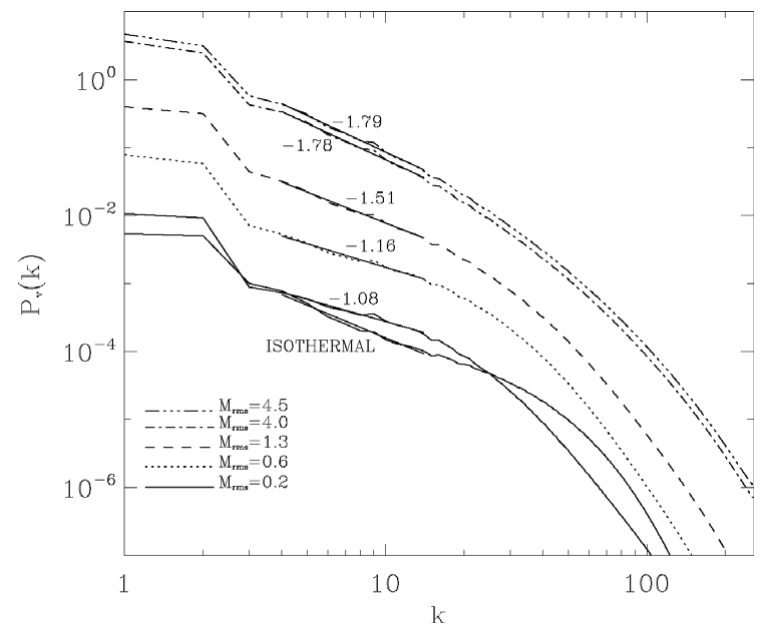

Figure 2. Velocity power spectra $P_{v}$ for simulations with different $M$. For $M=0.2$ the spectra resulting from an isothermal run is also displayed. Solid lines represent least-squares fits over the range $4 \leqslant k \leqslant 14$ and numbers indicate the slopes of these fits.

In conclusion, the presence of TI does significantly affect the statistics of turbulence in thermally bi-stable flows. Different turbulence conditions, in particular different Mach numbers, can lead to considerably different slopes of the density and the velocity power spectrum of the atomic interstellar gas.

\section{References}

Elmegreen, B. G., Scalo J. 2004, ARAA, 42, 211

Field, G. B. $1965, A p J, 142,531$

Gazol, A. \& Kim, J. 2010 ApJ, 630, 911

Kim, J. \& Ryu, D. 2005, ApJ, 630, L45

Scalo, J. \& Elmegreen, B. G. 2004, ARAA, 42, 275 